\title{
LA CONSERVACIÓN DE LOS INVERTEBRADOS EN ESPANA: UNA PERSPECTIVA HISTÓRICA
}

\section{J. R. Verdú, E. Galante y C. Numa}

\author{
Instituto de Biodiversidad CIBIO, Universidad de Alicante \\ jr.verdu@ua.es
}

\section{INTRODUCCIÓN}

Los Invertebrados superan el millón de especies descritas, lo que constituye aproximadamente el $65 \%$ de todos los organismos vivos conocidos y el $77 \%$ de las especies animales. Este elevado número de especies, unido al importante papel que desempeñan en los ecosistemas, no se corresponde con el escaso protagonismo que tradicionalmente han tenido en las políticas de conservación del medio, y su utilización en los estudios de ordenación del territorio ha sido prácticamente nula. Los invertebrados son organismos que encontramos interviniendo en los distintos niveles de los procesos ecológicos y por ello su conservación debiera recibir una atención prioritaria por parte de las administraciones e instituciones implicadas en la conservación de la Naturaleza.

Quizá la razón de esta falta de atención podemos encontrarla en la dificultad que muchas veces presenta su estudio, la falta de conocimiento taxonómico en algunos grupos, el desconocimiento de la biología $\mathrm{y}$ de los requerimientos ambientales de gran parte de las especies, así como la falta de información biogeográfica en muchas de ellas.
Sin embargo, paradójicamente muchos grupos de invertebrados han sido utilizados durante las últimas décadas como base de estudios de biodiversidad dirigidos a evaluar el estado conservación del medio, o bien como instrumentos para establecer áreas de conservación. Los estudios de biodiversidad llevan implícitos análisis a diferentes escalas que van desde los genes hasta los ecosistemas que conforman paisajes como unidades regionales en las que la acción humana cobra su mayor relevancia. Conservar los invertebrados, sus hábitats y los ecosistemas de los que forman parte, es conservar gran parte de la biodiversidad existente.

A lo largo de la relativamente corta historia de la Biología de la Conservación, la protección ha sido dirigida en mayor medida hacia dos unidades bien definidas: la especie y el hábitat (representado por un área concreta), pero la dificultad que entraña en ocasiones el estudio de las especies de invertebrados, ha hecho que raramente sean tomadas en consideración.

Si bien es cierto que para conocer la diversidad biológica que existe en un lugar, lo más conveniente sería llevar a cabo un inventario exhaustivo (NOSS, 1990), en el caso de los invertebrados existe una gran 
dificultad en realizar este proceso, y en particular de desarrollarlo en los cortos plazos de tiempo que requieren la toma de decisiones para proteger un área determinada (STORK et al., 1996). El resultado es que en raras ocasiones los invertebrados tienen protección legal y que las áreas naturales protegidas no se establecen tomando en consideración las especies del grupo. Otro problema ańadido es que en muchas ocasiones se protege lo que "gusta" desde un punto de vista antropocéntrico, sin tener en consideración los numerosos estudios científicos que demuestran el mayor interés de otras especies que suelen presentar un mayor grado de amenaza. En el caso de los invertebrados ocurre esta tendencia, por lo que no es de extrañar que los grupos más llamativos o de mayor belleza hayan tenido una cierta preferencia en los planes de conservación. Esto explica que el primer invertebrado protegido en el año 1835 fuese el lepidóptero Parnassius apollo en el Estado de Baviera (Alemania).

\section{LA PROTECCIÓN DE LOS INVERTEBRA. DOS EN ESPAÑA}

La Península Ibérica, y en particular España, es una de las áreas de mayor diversidad y endemicidad de toda Europa. Sin embargo, hasta hace unos años los invertebrados eran ignorados en cualquier plan de conservación y en la catalogación de especies amenazadas. En 1976 se publicó el primer Libro Rojo que consideraba a un grupo de artrópodos, los lepidópteros (VIEDMA \& GÓMEZ-BUSTILLO, $1976,1985)$ y casi diez años más tarde apareció el Libro Rojo de los Ortópteros ibéricos (Gangwere et al., 1985). Estas dos primeras referencias editadas por el desaparecido ICONA, actual Dirección General para la Biodiversidad del Ministerio de Medio Ambiente, son sin duda las primeras aportaciones dirigidas hacia la conservación de artrópodos en España. Posteriormente en 1992 se publicó la primera obra que recopilaba los distintos grupos de invertebrados de España protegidos por Convenios Internacionales (ROSAS et al., 1992).

En Octubre de 1993, la Asociación española de Entomología (AeE), organizó el primer simposio sobre la conservación de los artrópodos en España bajo el título International Conference on Arthropod
Management and Environmental Conservation. Como resultado de este simposio se elaboró el denominado Manifiesto de Calpe, ciudad en la que se celebró dicha reunión científica (JIMÉNEZ-PEIDRÓ \& MARCOS-GARCÍA, 1994).

De acuerdo con las propuestas de dicho manifiesto, la $A e E$ inició en 1995 la elaboración de una serie de fichas de especies que constituyeron la base inicial de lo que diez años más tarde iba a ser la Lista Roja de Artrópodos de España. Como parte de este proceso inicial, en Junio de 1995 la $A e E$ estableció un acuerdo de colaboración con la Dirección General de Conservación de la Naturaleza del Ministerio de Medio Ambiente, con el fin de designar los hábitats de las especies de artrópodos incluidas en la Directiva Europea de Conservación de los hábitats naturales y de la Fauna y Flora silvestre (Directiva 92/43/CEE) conocida como "Directiva Hábitat" y cuyo resultado fue el inventariado de las especies de artrópodos incluidas en el anexo 2 de la Directiva Hábitat (Figura 1) (GALANTE \& VERDÚ, 2000).

La Directiva Hábitat, si bien ha constituido una importante herramienta para la conservación de la biodiversidad europea, no obstante no incluye las especies ibéricas y macaronésicas más singulares y realmente interesantes bajo el punto de vista de la conservación. Por ello, una vez concluido el proyecto sobre los Artrópodos de la "Directiva Hábitat", el Centro Iberoamericano de la Biodiversidad (CIBIO) de la Universidad de Alicante en colaboración con la $A e E$, elaboró la propuesta de especies de artrópodos que debían incluirse en el Catálogo Nacional de Especies Amenazadas (Real Decreto 439/1990, de 30 de Marzo) referente a la Ley 4/1989, de 27 de Marzo, de Conservación de Espacios Naturales y de la Flora y Fauna Silvestres. Esta propuesta comprendía más de 100 fichas de especies clasificadas en las distintas categorías de amenaza contempladas por la Ley 4/89. En total se propusieron un total de 112 especies, de las cuales 18 se consideraban dentro de la categoría En Peligro de Extinción, 24 especies se propusieron como Vulnerables, y 61 especies se consideraron Sensibles a la alteración de su Hábitat, además de una especie incluida en el Convenio de Berna y 8 especies incluidas en la "Directiva Hábitat" únicas actualmente incluidas en el Catálogo Nacional de Especies Amenazadas (BOE no 148, 22 de Junio de 


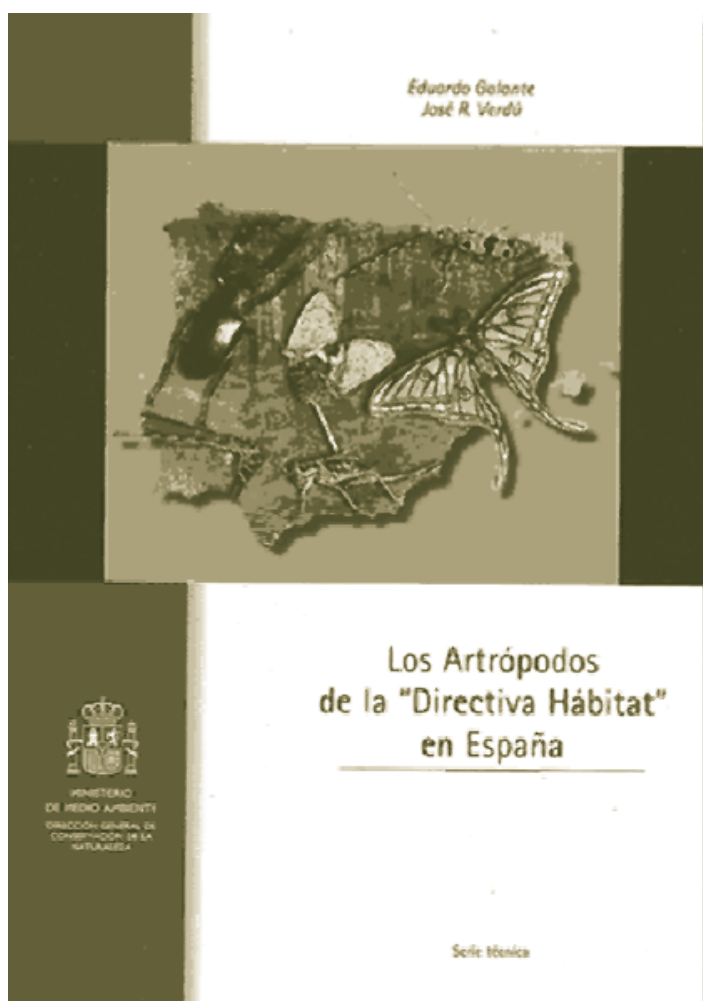

Figura 1. Los artrópodos de la "Directiva Hábitat" en España recopiló el inventariado de todas las especies presentes en el anexo número 2 de la presente Directiva Europea.

1999). A pesar del gran esfuerzo en la elaboración de estas listas de especies, hasta el momento únicamente han sido incluidas en el Catálogo Nacional de Especies unas pocas mientras que el resto, las más representativas de nuestro Patrimonio Natural, han sido olvidadas y en ocasiones no aceptadas hasta la fecha.

Igualmente, desde la Sociedad Española de Malacología se recopiló la información sobre 151 especies de moluscos, tanto marinos como dulceacuícolas y terrestres, cuyo estado de conservación se consideró amenazado. Este estudio de especies de moluscos amenazados (GÓMEZ et al., 2001) pretendía informar a las autoridades de Medio Ambiente sobre posibles candidatos para ser incluidos en futuras versiones del Catálogo Nacional de Especies Amenazadas.

Estas listas de especies han constituido posteriormente la base para la elaboración del Libro Rojo de invertebrados de España (Figura 2). Este proyecto fue desarrollado por el CIBIO en colaboración con la $A e E$ y la $S E M$ y se llevó a cabo de acuerdo con los criterios de UICN (categorías para las Listas Rojas versión 3.1), que considera con distinto grado, la rareza de la especies, la peculiaridad de los hábitats donde viven el grado de amenaza de los mismos, así como el estado de sus poblaciones.

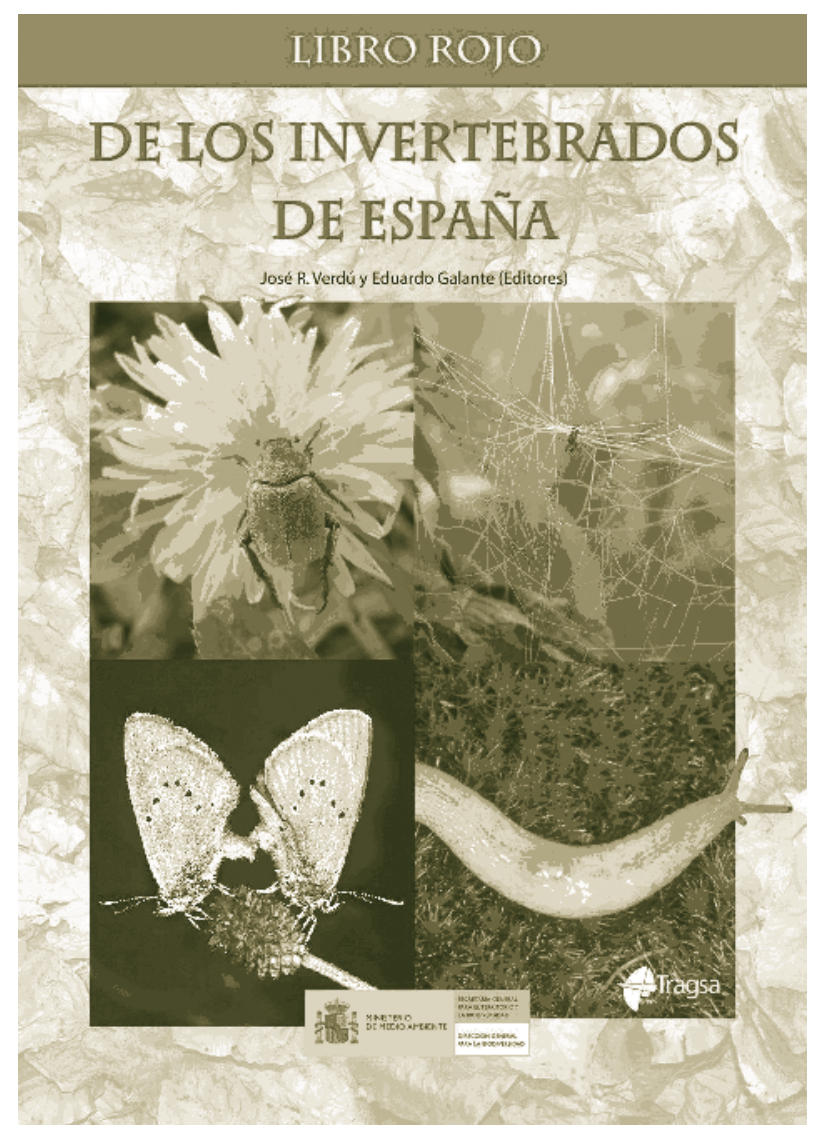

Figura 2. En 2006 se publica el Libro Rojo de los invertebrados de España.

El resultado de todo este proceso ha sido la edición del Libro Rojo de invertebrados de España (VERDÚ \& GALANTE, 2006), en el que han participado, a distintos niveles, cerca de 500 investigadores y que incluye 272 especies que presentan alguna de las categorías de amenaza contempladas por la UICN. En el caso de los artrópodos, las especies amenazadas ocupan una alta diversidad de hábitats, siendo los más representados los medios acuáticos y riparios (30\% aprox.), y el matorral mediterráneo en 
sentido amplio (19\%), que por primera vez cobra importancia frente a los hábitats típicos de bosque. Dentro de los hábitats de bosque, destacamos la mayor representatividad del bosque mediterráneo compuesto principalmente por quercíneas (6\%), frente a los bosques de especies caducifolias típicamente eurosiberianas, como es el caso de los hayedos (4\%). Por último, cabe resaltar la existencia de especies de artrópodos amenazadas características de hábitats que antes del Libro Rojo de Invertebrados de España no estaban bien representados y que se caracterizan por su gran vulnerabilidad y fragilidad como las especies propias de ambientes semidesérticos, esteparios, matorral mediterráneo, sistemas dunares, medios cavernícolas, entre otros. En el caso del matorral mediterráneo, especialmente los situados cerca de la costa, representan uno de los hábitats más amenazados por el aumento incontrolado de la urbanización y el abandono de las actividades agropecuarias que durante milenios han mantenido una estructura del paisaje que actualmente está en crisis. Son numerosas las especies de invertebrados propios de los hábitats costeros que se pueden ver amenazados por los cambios de usos de suelo y la fuerte presión urbanística, muchas de las cuales ya han sido incluidas en el Libro Rojo con alguna categoría de amenaza.

El Libro Rojo de los Invertebrados de España ha sido un primer paso importante para proteger los invertebrados, pero no constituye un fin en si mismo, ya que la conservación de las especies aisladas no es una política correcta para conservar la biodiversidad. Sólo a través de la conservación de los hábitats y teniendo en cuenta los procesos ecológicos en los que intervienen las distintas especies, podremos llegar a una efectiva conservación de la Naturaleza. El Libro Rojo por tanto debemos entenderlo como un documento dinámico en el tiempo y sujeto a sucesivas actualizaciones que optimicen su utilidad como herramienta en la Conservación de la Biodiversidad. En este sentido, los datos que contiene esta publicación constituyen una buena

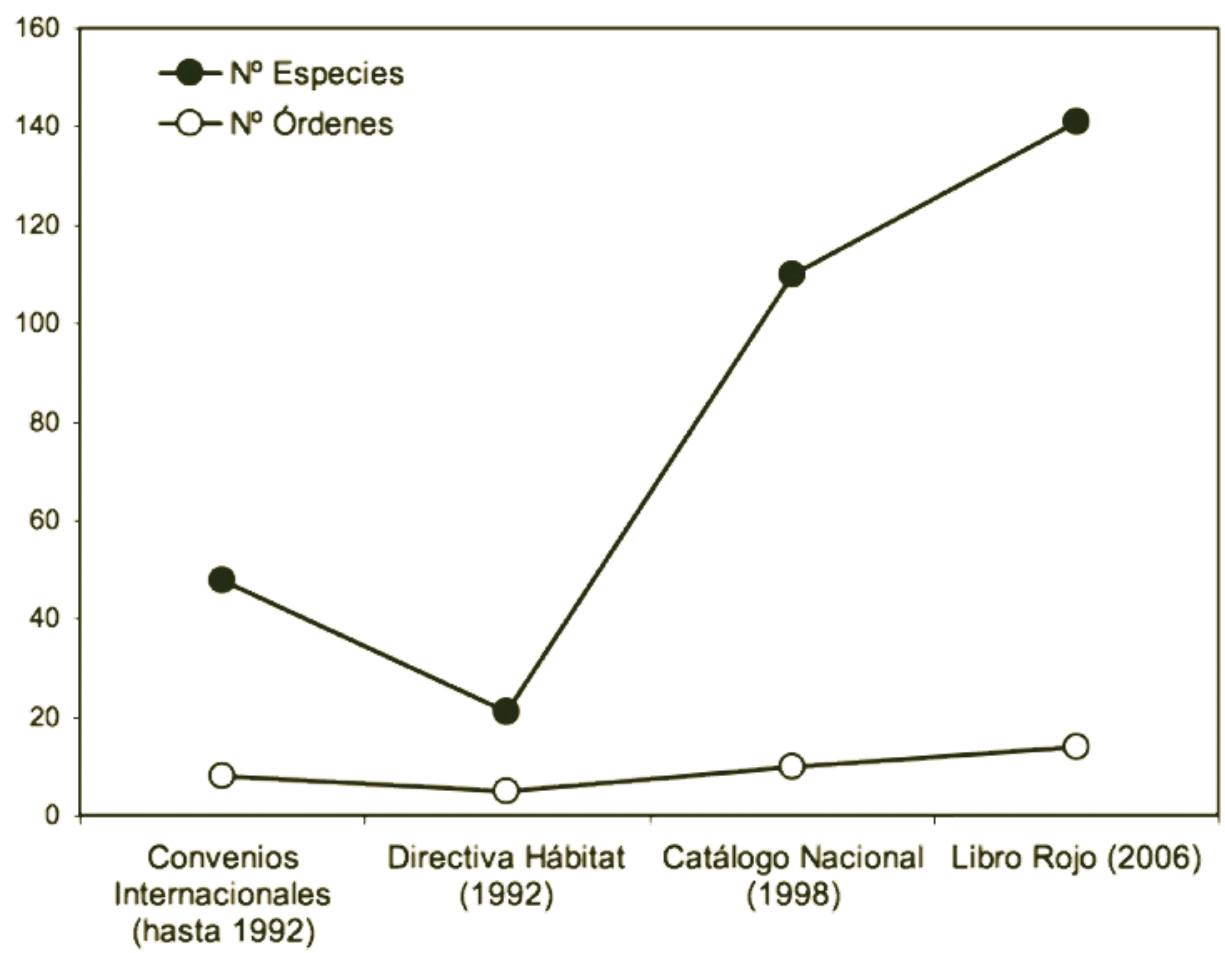

Figura 3. Evolución del número de artrópodos considerados ( $n^{o}$ de especies y $n^{o}$ de órdenes) a lo largo de la historia de la protección de especies en España. Fuente: Libro Rojo de invertebrados de España. 


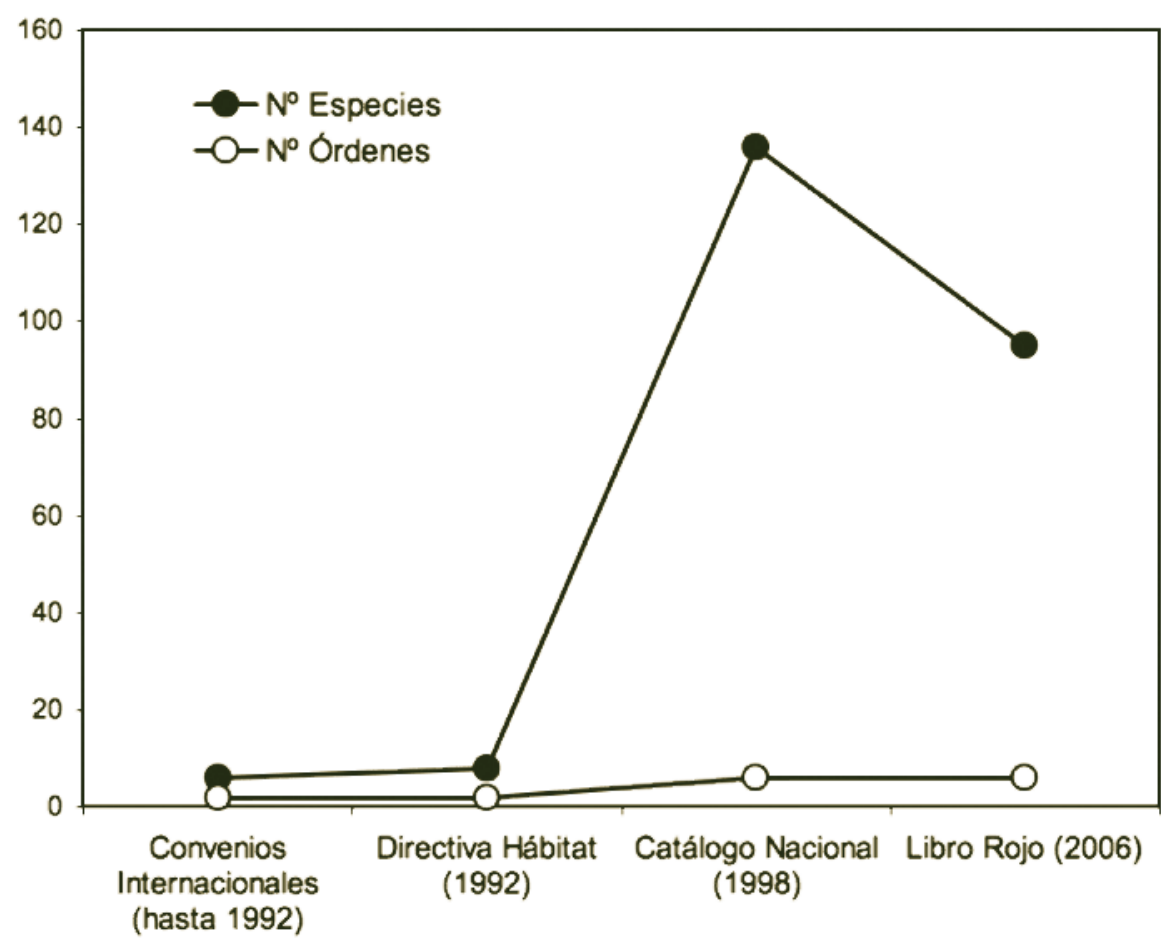

Figura 4. Evolución del número de moluscos considerados ( $n^{\circ}$ de especies y $n^{\circ}$ de órdenes) a lo largo de la historia de la protección de especies en España. Fuente: Libro Rojo de invertebrados de España.

aproximación al estado de conservación y grado de amenaza de este grupo animal y la elaboración de esta Lista Roja, siguiendo criterios de la UICN, debe hacernos reflexionar sobre la importancia de los invertebrados, tan olvidados en ocasiones aun representando el mayor porcentaje de diversidad de nuestro patrimonio natural. Desde el punto de vista histórico podemos destacar el incremento en el conocimiento de los invertebrados amenazados en España que se ve reflejado en el aumento del número de especies y órdenes considerados en los sucesivos trabajos realizados (véase Figuras 3 y 4).

Por último sólo queda reseñar que el Libro Rojo de los Invertebrados de España constituye en este momento la base de partida del proyecto Atlas de los Invertebrados amenazados de España que en estos momentos se ha iniciado desde el CIBIO, impulsado por la Dirección General para la Biodiversidad del Ministerio de Medio Ambiente, y nuevamente con la colaboración de la $A e E$ y de la $S E M$. Este Atlas aborda el estudio detallado y actualizado de distribución de las especies incluidas en el Libro
Rojo, revisando en su caso la categoría que se le ha atribuido a tenor de los resultados de las prospecciones de campo y facilitando las distribuciones con una precisión mínima de $10 \mathrm{~km}$, siendo en ocasiones de $1 \mathrm{~km}$. En esta cartografía de detalle se adjuntan los grados de amenaza de cada una de las poblaciones estudiadas en cada cuadrícula de estudio.

\section{AGRADECIMIENTOS}

Tenemos que agradecer a todos los socios de la $A e E$ y de la $S E M$ por el trabajo desarrollado hasta la fecha. Destacamos el buen trabajo de equipo realizado con Elena Bermejo y Fernando Corrales (Área de Medio Ambiente, Tragsa) y especialmente el incondicional apoyo de Cosme Morillo durante todos estos años. Por último, queremos dar las gracias a la Dirección General para la Biodiversidad del Ministerio de Medio Ambiente por apostar cada vez más por los invertebrados como grupo de estudio para la conservación de la Biodiversidad en España. 


\section{REFERENCIAS}

GALANTE, E. \& J.R. VERDÚ. 2000 (eds.). Los Artrópodos de la "Directiva Hábitat" en España. Organismo Autónomo de Parques Nacionales (MMA), serie técnica. $247 \mathrm{pp}$.

GANGWERE, S.K., M.G. DE VIEDMA \& V. LLORENTE. 1985. Libro Rojo de los Ortópteros Ibéricos. Monografías no 41. ICONA, Madrid, $91 \mathrm{pp}$.

GÓMEZ, B., MORENO, D., ROLÁN, E. ARAUJO, R. \& ÁLVAREZ R. M. (eds.). 2001. Protección de moluscos en el Catálogo Nacional de especies amenazadas. Reseñas Malacológicas No XI. Sociedad Española de Malacología. 286 pp. JIMÉNEZ-PEYDRÓ, R. \& M.A. MARCOSGARCÍA. 1994. Environmental management and Arthropod conservation. Asociación española de Entomología. 179 pp.
NOSS, R.F. 1990. Indicators form monitoring of biodiversity: a hierarchical approach. Conservation Biology, 4: 355-364.

ROSAS, G., M.A. RAMOS \& A. GARCÍA VALDECASAS. 1992. Invertebrados españoles protegidos por convenios internacionales. ICONA. 250 pp.

STORK, N.E., M.J. SAMWAYS \& H.A.C. EELEY. 1996. Inventoring and Monitoring biodiversity. Trends in Ecology and Evolution, 11: 15-16.

VERDÚ, J.R. \& E. GALANTE. 2006. Libro Rojo de los invertebrados de España. Ministerio de Medio Ambiente, Madrid. 411 pp.

VIEDMA, M.G. \& M.R. GÓMEZ BUSTILLO. 1976. Libro rojo de los Lepidópteros ibéricos. ICONA., Madrid, 120 pp.

VIEDMA, M.G. DE \& M.R. GÓMEZ BUSTILLO. 1985. Revisión del libro rojo de los lepidópteros ibéricos. Monografías ICONA 42, Madrid, $71 \mathrm{pp}$. 